\title{
Active tracked intramyocardial catheter injections for regenerative therapy with real-time MR guidance: feasibility in the porcine heart
}

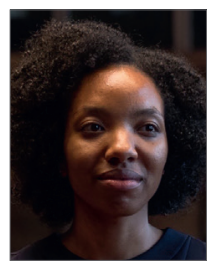

Cheyenne C.S. Tseng ${ }^{1,2}$, MD; Steven Wenker ${ }^{1}$, MD; Maarten H. Bakker ${ }^{3}, \mathrm{PhD}$;

Adriaan O. Kraaijeveld ${ }^{1}$, PhD; Patricia Y.W. Dankers ${ }^{3}$, PhD; Peter R. Seevinck ${ }^{4}$, PhD;

Jouke Smink 5 , MsC; Scott Kimmel ${ }^{6}, \mathrm{MsC}$; Frebus J. van Slochteren ${ }^{1,7}, \mathrm{PhD}$;

Steven A.J. Chamuleau ${ }^{1,2 *}, \mathrm{MD}, \mathrm{PhD}$

1. Division of Heart and Lungs, Department of Cardiology, University Medical Center Utrecht, Utrecht, the Netherlands;

2. Netherlands Heart Institute (NHI), Utrecht, the Netherlands; 3. Institute for Complex Molecular Systems, Department of

Biomedical Engineering, Laboratory of Chemical Biology, Eindhoven University of Technology, Eindhoven, the Netherlands;

4. Image Sciences Institute, University Medical Center Utrecht, Utrecht, the Netherlands; 5. Philips Research Laboratories, Best,

the Netherlands; 6. Imricor Medical Systems, Burnsville, MN, USA; 7. CART-Tech B.V., Utrecht, the Netherlands

C.C.S. Tseng and S. Wenker contributed equally to this work as co-first authors.

F.J. van Slochteren and S.A.J. Chamuleau contributed equally to this work as co-senior authors.

This paper also includes supplementary data published online at: https://eurointervention.pcronline.com/doi/10.4244/EIJ-D-17-01081

\section{Introduction}

The use of magnetic resonance imaging (MRI) for guidance of interventional procedures (iMRI) has distinct advantages over fluoroscopy. MRI lacks ionising radiation, has superb soft tissue contrast and provides functional data. Although iMRI is not suitable for all patient categories (e.g., cardiac devices), its potential has already been demonstrated for various interventions ${ }^{1,2}$.

Within the field of cardiac regenerative therapy there is a need for improved delivery of therapies ${ }^{3}$. Currently, regenerative therapy is applied via coronary infusion or electro-anatomical mapping-guided injections. Because late gadolinium (LG)-MRI is the gold standard for infarct assessment and allows real-time soft tissue imaging, we hypothesise that iMRI-guided intramyocardial injections could generate a unique insight into the injection procedure and boost effectiveness of regenerative therapies.

For visualisation of catheters during iMRI procedures, passive visualisation or active tracking can be utilised.

\section{PASSIVE VISUALISATION}

Passive catheter visualisation relies on the intrinsic properties of catheter materials to create MR-contrast and real-time images are acquired with a high temporal and spatial resolution. This provides visualisation of the catheter trajectory in combination with live anatomy. A disadvantage of passive visualisation is that the imaging plane does not automatically adjust to the catheter position and has to be adjusted manually.

\section{ACTIVE TRACKING}

Active tracking utilises a special tracking MR sequence and receiver coils (tracking coils) that are incorporated in the catheter. This allows the catheter position to be deduced from the signal received from the tracking coils. Because the tracking sequence does not allow simultaneous creation of live images, a high-resolution 3D anatomical "roadmap" scan is acquired prior to the intervention to visualise anatomy.

With active tracking the iSuite interventional software:

- registers the catheter location based on signals from the tracking coils,

- selects imaging planes that contain the catheter location,

- renders a 3D model of the catheter in conjunction with images.

Additional information (such as surface potentials) can be projected onto the endomyocardial surface' ${ }^{1}$. Automatic plane

*Corresponding author: Division of Heart and Lungs, Department of Cardiology, University Medical Center Utrecht, Heidelberglaan 100, Room E.04-511, Post office box 85500, 3508 GA Utrecht, the Netherlands. E-mail: s.a.j.chamuleau@umcutrecht.nl 
adjustment makes active tracking more intuitive than passive visualisation to guide towards target locations. Passive visualisation is mainly used at specific moments during the procedure (for example, crossing the aortic valve).

\section{Methods}

A short moving image of the procedure (Moving image 1) is provided as supplementary data.

\section{MR-GUIDED INJECTION PLATFORM}

Real-time MR-guided interventions require specific tools and technologies. Within this project we have combined three essential components:

1. an interventional MRI set-up with active tracking capability,

2. a steerable, trackable injection catheter,

3. an MR-visible injectable biomaterial.

\section{ANIMALS}

Experiments were performed in seven female Landrace pigs $( \pm 70 \mathrm{~kg})$.

\section{INTERVENTIONAL SUITE AND TRACKING INTERFACE}

The real-time image guidance platform iSuite (Philips Research, Hamburg, Germany) was installed on a 1.5T MR scanner (Ingenia; Philips Healthcare, Best, the Netherlands) at the Utrecht University Veterinary Department. Figure 1A shows a schematic overview of our set-up.

\section{MR COMPATIBLE INJECTION CATHETER}

A trackable, steerable injection catheter (Imricor Medical Systems, Burnsville, MN, USA) was adapted from an Imricor ablation catheter ${ }^{1}$. Two tracking coils are integrated into the catheter tip. Catheter specifications are summarised in Table 1 and Figure 1B.

\section{UPy-PEG HYDROGELATOR}

For injections, a UPy-based pH-responsive hydrogel, crosslinked with a contrast-enhancing agent UPy-Gd ${ }^{4}$, was used.

\section{Results}

\section{ACCESSING THE LEFT VENTRICLE}

The left ventricle (LV) was approached retrogradely via the right femoral artery. To pass the aortic valve without damaging the injection catheter, we created a long introduction sheath that could be placed through the aortic valve. The sheath was put in place over an actively trackable introducer catheter designed for this purpose. Under active tracking, the catheter was advanced retrogradely towards the aortic valve. When the catheter had passed the aortic valve, we advanced the sheath over the catheter and exchanged the introduction catheter for the injection catheter.

\section{NAVIGATION AND STEERING}

A 3D mesh of the LV was created from the roadmap scan (ITKSNAP software). The sample frequency of 5-10 Hz during active

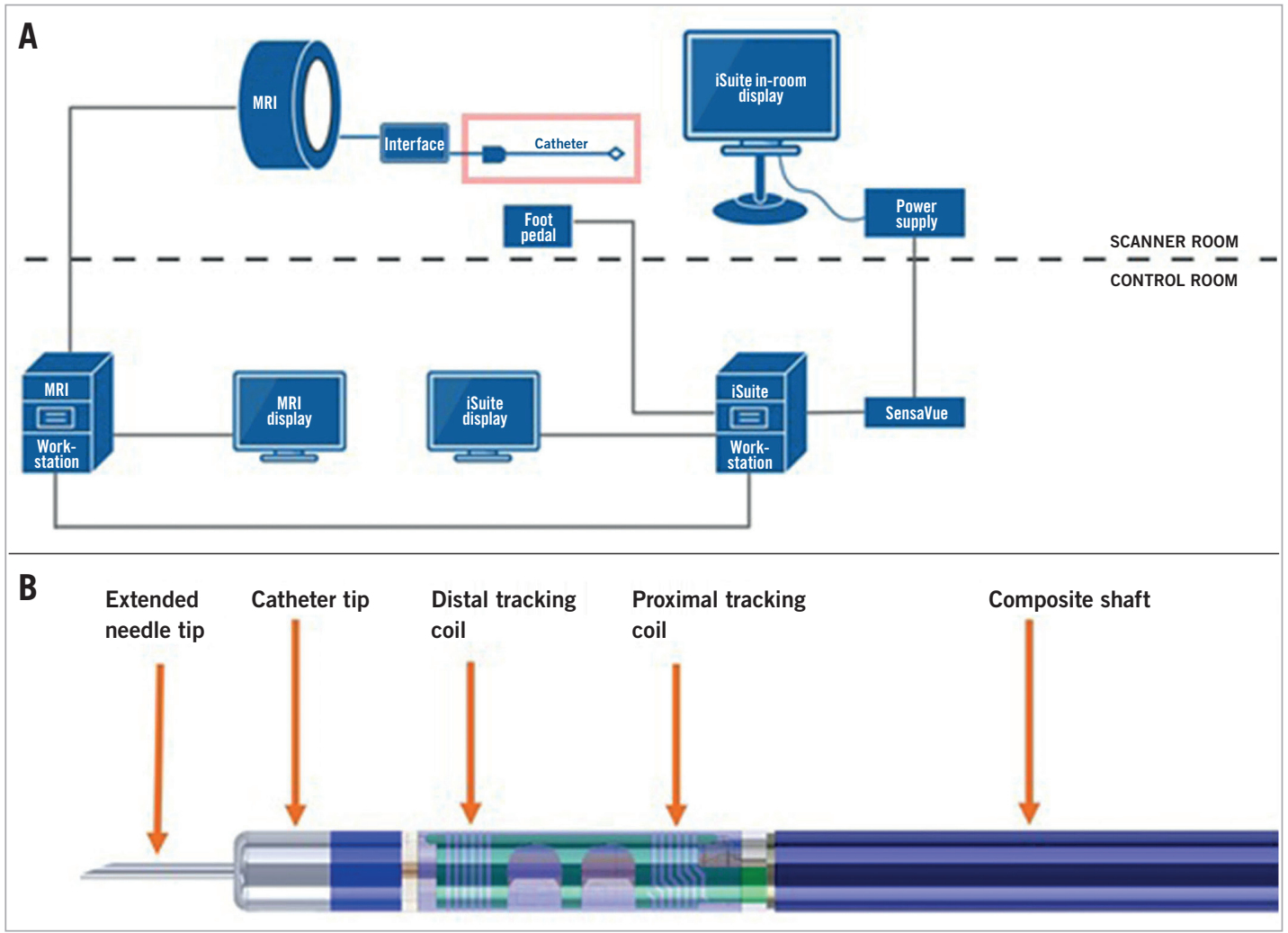

Figure 1. Schematic overviews of our interventional MR set-up and the injection catheter. A) Interventional MR set-up. B) Injection catheter. 
Table 1. Catheter specifications.

\section{Catheter specifications}

\begin{tabular}{|c|c|c|}
\hline \multicolumn{2}{|c|}{ Outer diameter } & $9 \mathrm{Fr}$ \\
\hline \multicolumn{2}{|l|}{ Length } & $110 \mathrm{~cm}$ \\
\hline \multirow[t]{3}{*}{ Deflection } & Deflection & Unidirectional $180^{\circ}$ \\
\hline & Inner diameter & $32 \mathrm{~mm}$ \\
\hline & Mechanism & Pullwire \\
\hline \multirow[t]{7}{*}{ Needle } & Length & $145 \mathrm{~cm}$ \\
\hline & Extension & Adjustable \\
\hline & \multirow[t]{2}{*}{ Material } & Nitinol (distal $13 \mathrm{~cm}$ ) \\
\hline & & Polymer (rest) \\
\hline & \multirow[t]{2}{*}{ Inner diameter } & Nitinol: 0.25 mm (30 gauge) \\
\hline & & Polymer: $0.64 \mathrm{~mm}$ \\
\hline & Bevel & $20^{\circ}$ straight bevel \\
\hline \multicolumn{2}{|l|}{ Tracking } & 2 distal tracking coils $6 \mathrm{~mm}$ apart \\
\hline
\end{tabular}

\section{Introducer catheter}

\begin{tabular}{|l|l|}
\hline Outer diameter & $9 \mathrm{Fr}$ \\
\hline Length & $110 \mathrm{~cm}$ \\
\hline Material & Polymer \\
\hline Tracking & 2 distal tracking coils $6 \mathrm{~mm}$ apart \\
\hline Introducer sheath &
\end{tabular}

\begin{tabular}{|l|l|}
\hline Outer diameter & $14.5 \mathrm{Fr}$ \\
\hline Inner diameter & $9 \mathrm{Fr}$ \\
\hline Length & $110 \mathrm{~cm}$ \\
\hline Material & Polymer \\
\hline
\end{tabular}

tracking allowed intuitive navigation towards specific targets (Figure 2, Moving image 1). When a target was reached, passive visualisation was used to confirm wall contact. If needle extension triggered a premature ventricular contraction (PVC), an injection was performed. Three to five injections $( \pm 200 \mu \mathrm{l})$ were performed at diverse locations. Passive visualisation allowed visualisation of the injected material during injection. For confirmation we performed a post-injection scan after each injection. Table 2 contains details on the MR sequences used.

\section{SAFETY}

No safety issues occurred.

Table 2. Imaging protocol.

\begin{tabular}{|c|c|}
\hline & Imaging protocol \\
\hline Roadmap scan & $\begin{array}{l}\text { Respiratory- and cardiac-gated fat-suppressed } \\
\text { T1-weighted 3D whole heart bTFE }\end{array}$ \\
\hline & $\begin{array}{l}\text { TE } 2.3 \mathrm{~ms} / \mathrm{TR}: 4.7 \mathrm{~ms} / \text { voxel size } 1.5 \mathrm{~mm}^{3} \\
\text { (isotropic) reconstructed to } 1.15 \times 1.15 \times 1.5 \mathrm{~mm}^{3}\end{array}$ \\
\hline Passive tracking & $\begin{array}{l}2 \mathrm{D} \text { balanced } \mathrm{bTFE} \\
\text { frame rate: } 3 \mathrm{~Hz}\end{array}$ \\
\hline Active tracking & 1D gradient echo projections, frame rate $10 \mathrm{~Hz}$ \\
\hline Post-injection & $\begin{array}{l}\text { 2D T1W TFE with PSIR reconstruction sequences } \\
\text { TE } / T R=3.0 \mathrm{~ms} / 6.1 \mathrm{~ms} \text {; flip angle: } 25 \text {; resolution } \\
1.6 \times 2.0 \mathrm{~mm} \text { reconstructed to } 0.9 \times 0.9 \mathrm{~mm} \text {, slice } \\
\text { thickness } 10 \mathrm{~mm}\end{array}$ \\
\hline Ex vivo & $\begin{array}{l}\text { 3D T1W TFE inversion recovery (LGE sequence) } \\
\text { resolution } 2 \times 3.3 \times 5 \mathrm{~mm} \text { reconstructed to } \\
1.23 \times 1.23 \times 5 \mathrm{~mm}\end{array}$ \\
\hline
\end{tabular}

\section{Discussion}

The MIGRATE (MrI Guided RegenerAtive ThErapy) consortium is a combined scientific and industrial effort to establish an MRIguided intramyocardial injection platform. By collaborating within the MIGRATE consortium we were able to combine state-of-theart interventional imaging hardware, software, MRI-compatible catheters, and biomaterials. In this pilot study, we demonstrated the feasibility of intramyocardial injections of an MRI-visible biomaterial by interventional MRI using a combination of active tracking and passive visualisation.

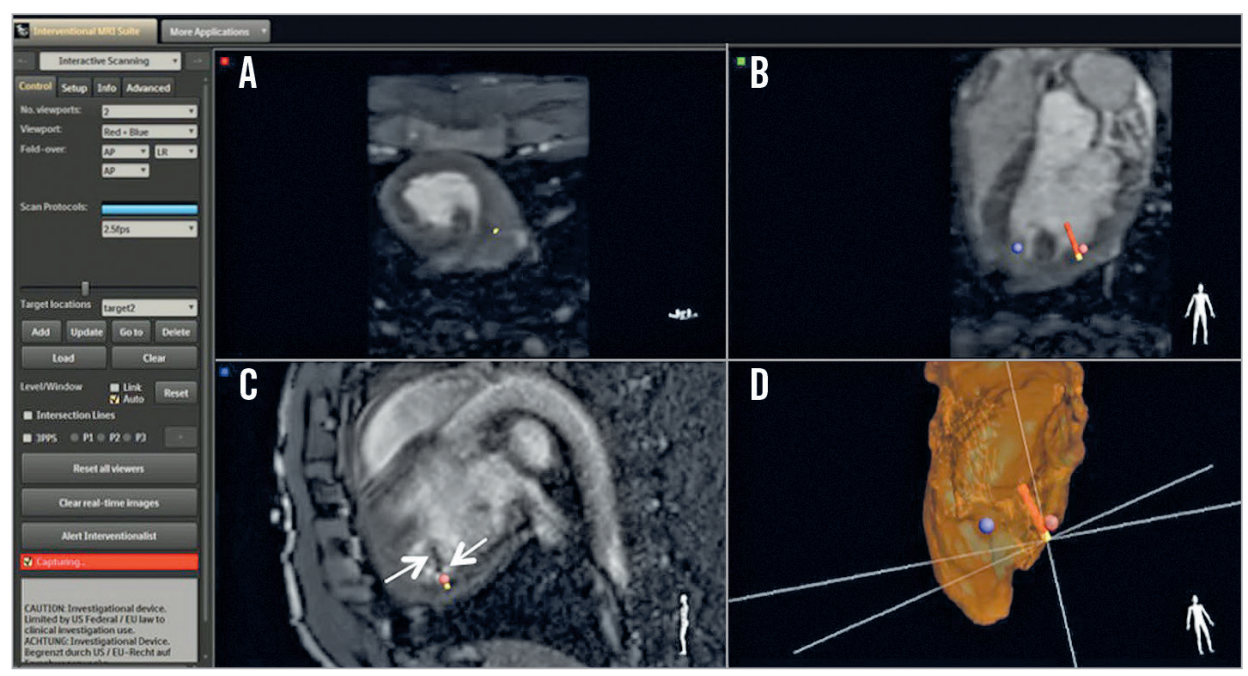

Figure 2. Suite screenshot with three orthogonal views and 3D LV endocardial segmentation. A) - C) Three orthogonal views. D) $3 D$ LV endocardial segmentation. 
The active tracking coils require a relatively large diameter catheter, that is harder to pass through the aortic valve. We solved this issue by creating a purpose-made introduction sheath that provides easy access to the left ventricle. This allows easy removal and reintroduction of catheters, for example in order to flush the catheter.

\section{Limitations}

Although the number of experiments is limited, we show that the combination between active tracking and passive visualisation allows delivery to specifically targeted areas. The UPy-PEG hydrogel is promising in order to improve retention of biological therapies: by combining the gel with a gadolinium-based contrast agent we were able to visualise the gel in real time. The immediate visualisation of the injected deposit is a unique feature of our setup and provides a new parameter to improve regenerative therapy.

The first steps towards cardiac application of interventional MR are already performed in the setting of ablations and biopsies ${ }^{1,2}$. Eventually, the use of MR guidance may help to shorten procedures, lower risks and improve injection accuracy and retention of regenerative therapies. Although both interventional MRI and regenerative therapy are still niche applications, with the combination of these tools we aim to add to the clinical effectiveness of cardiac regenerative therapy.

\section{Conclusion}

Within this project we designed, developed and tested the catheter on tracking, accessing the left ventricle and use in combination with a biomaterial. For the first time, real-time MR-guided intramyocardial injections have been performed with combined active and passive catheter tracking. The use of an MR-visible hydrogel allows in vivo tracking of injections. This novel delivery strategy may constitute an important step forward with regard to the accuracy, retention and mechanism of cardiac regenerative therapies.

\section{Impact on daily practice}

Interventional MRI is a steadily advancing technique that offers important advantages over fluoroscopy-guided procedures. This study adds intramyocardial injections to the palette of applications for this technique. Our work enables optimal injection guidance and visualisation and brings improved delivery, guided by iMRI, one step closer to clinical application. The next step towards a first-in-man application is validation of the technique in a porcine infarction model and assessing its accuracy in targeting the infarction border zone.

\section{Acknowledgements}

This work was performed at the UMC Utrecht with support from partners of the MIGRATE consortium: Philips Research, University of Technology Eindhoven, CART-Tech, Imricor Medical Systems, Nano4Imaging, Cook Medical, Personal Space Technologies, Netherlands Heart Institute and the Dutch Heart Foundation.

\section{Funding}

This work was conducted with financial support from the NHS, ICIN-Netherlands Heart Foundation and partners of the MIGRATE consortium (within the TKI LSH framework).

\section{Conflict of interest statement}

S. Chamuleau and F. van Slochteren are co-founders of CARTTech B.V. The other authors have no conflicts of interest to declare.

\section{References}

1. Hilbert S, Sommer P, Gutberlet M, Gaspar T, Foldyna B, Piorkowski C, Weiss S, Lloyd T, Schnackenburg B, Krueger S, Fleiter C, Paetsch I, Jahnke C, Hindricks G, Grothoff M. Real-time magnetic resonance-guided ablation of typical right atrial flutter using a combination of active catheter tracking and passive catheter visualization in man: initial results from a consecutive patient series. Europace. 2016;18:572-7.

2. Rogers T, Ratnayaka K, Karmarkar P, Campbell-Washburn AE, Schenke WH, Mazal JR, Kocaturk O, Faranesh AZ, Lederman RJ. Real-time magnetic resonance imaging guidance improves the diagnostic yield of endomyocardial biopsy. JACC Basic to Transl Sci. 2016;1:376-83.

3. Tongers J, Losordo DW, Landmesser U. Stem and progenitor cell-based therapy in ischaemic heart disease: promise, uncertainties, and challenges. Eur Heart J. 2011;32;1197-206.

4. Bakker MH, Tseng CCS, Keizer HM, Seevinck PR, Janssen HM, Van Slochteren FJ, Chamuleau SAJ, Dankers PYW; MRI Visualization of Injectable Ureidopyrimidinone Hydrogelators by Supramolecular Contrast Agent Labeling. Adv Healthc Mater. 2018;7:e1701139.

\section{Supplementary data}

Moving image 1. Overview of our intramyocardial injection procedure.

The supplementary data are published online at: https://eurointervention.pcronline.com/ doi/10.4244/EIJ-D-17-01081 